\title{
Investigation of the hydration shell of a membrane in an open system molecular dynamics simulation
}

\author{
John Whittaker $\odot^{*}$ and Luigi Delle Site $\odot^{\dagger}$ \\ Freie Universität Berlin, Institute of Mathematics, Arnimallee 6, 14195 Berlin, Germany
}

(Received 21 August 2019; published 13 November 2019)

\begin{abstract}
We analyze structural properties of hydration for a biological membrane with the open boundary adaptive resolution molecular dynamics approach in its latest version. A large region of noninteracting pointlike particles (tracers) acts as a reservoir of molecules and energy for the atomistically resolved membrane-water interface. The drastic simplification of the environment and its simple coupling to the high-resolution region has been successfully tested in a previous work on pure liquid water, and this work represents the method's first application to a system with high structural complexity. The successful results and novel methodology allow us to suggest a complementary view to the controversial question of the definition of the hydration shell for a membrane. The adaptive resolution technique allows for a physically well-founded statistical mechanics definition of the hydration shell by quantifying the minimal size of the region where the atomistic resolution of the molecules is strictly required while the rest of the system plays the role of a generic, structureless thermodynamic reservoir.
\end{abstract}

DOI: 10.1103/PhysRevResearch.1.033099

\section{INTRODUCTION}

\section{A. Hydration shell in biological membranes}

The water-water hydrogen bonding network deformed by the presence of polar groups, to which water molecules locally bond, characterizes the structural properties of hydration of a biological membrane [1]. In this context, the hydration shell plays a critical role in the appropriate function of the membrane, and according to an authoritative review in the field [2], its definition represents a nontrivial issue that can be a source of controversy. In molecular simulations, the concept of a system's hydration shell is usually defined in terms of empirical geometric criteria, such as the the first minimum of a radial distribution function [3]; however, such kinds of definitions do not provide precise details about the most relevant microscopic degrees of freedom that characterize interface properties. In a recent work on ionic liquids [4], our group has proposed a statistical mechanics definition of nanodroplets: the size of a droplet is defined as the size of the region where atomistic degrees of freedom are strictly required while the effect of the rest of the liquid can be modeled as a structureless, macroscopic thermodynamic reservoir. Such a definition highlights the relevance of the chemical molecular degrees of freedom and their range of action for a given structural property-they are sufficient to describe local liquid properties independently of any structural support from the bulk. In this view, we propose to apply the same criterion in the characterization of the hydration shell of a membrane,

\footnotetext{
*johnwhittake@zedat.fu-berlin.de

$\dagger$ luigi.dellesite@fu-berlin.de
}

Published by the American Physical Society under the terms of the Creative Commons Attribution 4.0 International license. Further distribution of this work must maintain attribution to the author(s) and the published article's title, journal citation, and DOI. that is, define the minimal interface region in which the atomistic description of water is mandatory, while bulk water can be represented by a generic reservoir of particles and energy at a given macroscopic thermodynamic condition. A definition as suggested here gives, for example, the advantage of designing interventions at the microscopic or molecular scale to obtain specific interface properties on demand without considering the details of the system on a large-scale besides its macroscopic thermodynamic state. In the past, similar concepts have been applied successfully to the analysis of the solvation shells of fullerene molecules [5] and of micelles [6].

\section{B. Definition of the relevant atomistic degrees of freedom via AdResS}

The implementation of the proposed criterion is achieved through a molecular dynamics approach that employs the adaptive resolution simulation technique (AdResS). In AdResS, the simulation box is divided into three regions [represented pictorially by Fig. 1(c)]: a region where molecules are resolved at atomistic level (AT), a region where molecules are represented by a simplified model without any chemical specificity (TR), and a coupling region $(\Delta)$ which acts as a buffer, allowing for the drastic change in resolution that occurs when a molecule passes from the TR region to the AT region (or vice versa). The $\Delta$ region and the TR region play the role of a large thermodynamic bath for the atomistic region. The minimal size of the atomistic region in AdResS which delivers structural properties faithfully reproducing those calculated in a full atomistic simulation of reference defines the region where the atomistic or chemical degrees of freedom are strictly required for the properties analyzed. The technical innovation of this work is the use of the most recent version of AdResS where the majority of the system's reservoir, TR, is represented by noninteracting, pointlike tracer particles with a direct coupling to the transition region $\Delta$ [7]. Given the molecular complexity of the system considered in this 


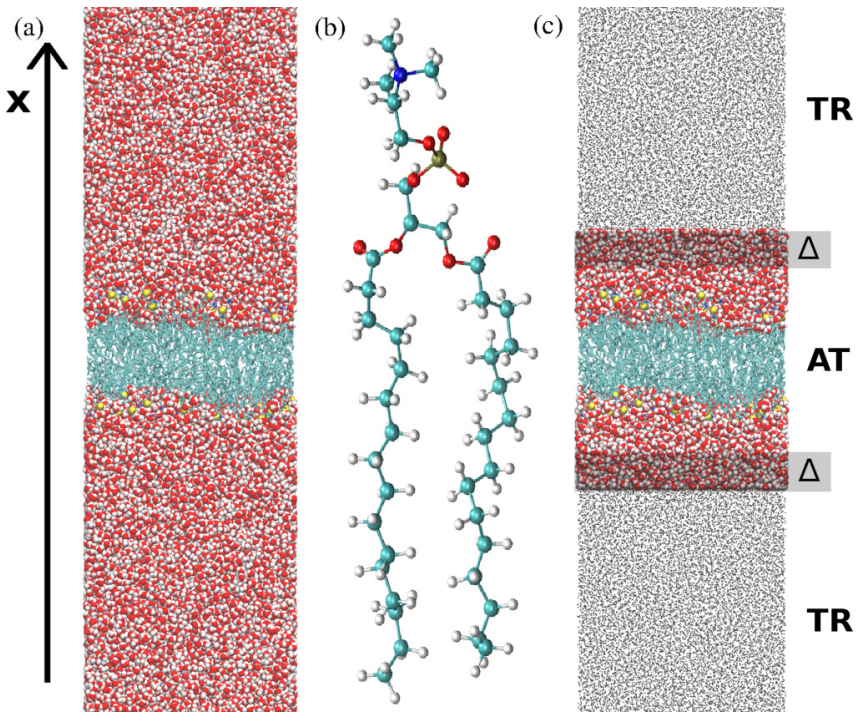

FIG. 1. (a) Full atomistic representation of the system. (b) Atomistic representation of the DPPC molecule. (c) AdResS simulation setup: the atomistically resolved DPPC bilayer is oriented in the $z-y$ plane and hydrated on top and bottom (along the $x$ axis) by atomistically resolved water (AT region). Beyond the AT region on both sides is the transition region $(\Delta)$ followed by the noninteracting tracer region (TR). DPPC alkane chains are represented in light blue while the negatively charged phosphorus and the positively charged nitrogen atoms in the DPPC head groups are represented in yellow and blue, respectively. Hydrating water molecules are shown in a space-filling representation and tracer particles are represented by black beads. Periodic boundary conditions are applied in $x, y$, and $z$.

work, the capability of the method to deliver satisfactory results is a technical verification of its capacity for simulating large complex molecular environments [8]. Here, we will consider a DPPC bilayer whose full atomistic system of reference consists of 180 (90 per leaflet) DPPC molecules [see Figs. 1(a), 1(b)]. The bilayer is then solvated with 52470 water molecules in a box of dimensions $33.4 \mathrm{~nm} \times 7.5 \mathrm{~nm} \times$ $7.5 \mathrm{~nm}$. The membrane is oriented in the $y-z$ plane with the normal along the $x$ axis. It must be reported that a first attempt of AdResS simulation of a membrane was performed in Ref. [9]. The results are encouraging and of high technical interest, but the overall conceptual approach is empirical and not yet properly formalized in a suitable statistical ensemble, while the methodology utilized in this paper is already conceptually well founded.

\section{Summary of the key aspects and organization of the paper}

In summary, this paper presents two different, equally relevant and intimately linked aspects: (1) The proof that our computational technique, guided by well-defined, strict physical criteria of statistical mechanics, properly describes a highly complex molecular system of primary relevance in current studies. From a technical as well as conceptual point of view, it is not a trivial result that the complex molecular architecture of a biological membrane embedded in a solvent can be accurately treated as an open system. (2) The previous point represents a key mandatory condition for the consequent physical analysis of the system in terms of an open boundary, solvated macromolecular structure embedded in an open environment with which energy and particles can be exchanged. A crucial conceptual point is that an open system analysis implies the possibility of determining, in a quantitative manner, the extension of the region of space where chemical or atomistic degrees of freedom play a relevant role, while outside a generic thermodynamic bath is sufficient. As a consequence, the approach allows us to offer a unique insight into the role of the hydrogen bonding of the solvent in the stability of the membrane. In turn, such a view provides complementary information to the discussion about the extent of the hydration shell of the membrane. Interestingly, our study agrees with results of previous work where it is shown that water properties are influenced by biomolecules for distances far beyond distances coinciding with the first minimum of radial distribution functions, a criterion often empirically chosen to determine the hydration layer.

The paper is organized as follows: The basic principles of AdResS are reported in the next section, which is followed by a section describing the results. First, we show that the necessary conditions for a physically consistent open system are satisfied for the minimal AT region. Next, the analysis of several structural properties confirms that the atomistic degrees of freedom in the minimal AT region are sufficient for a faithful reproduction of the same properties calculated in a fully atomistic system of reference. Finally, a discussion of the results and their relation to results of previous work by other groups is reported. An Appendix reports all technical details needed for the reproduction of the results shown.

\section{BASIC PRINCIPLES OF AdResS AND THE CRITERIA FOR AN OPEN SYSTEM}

The computational implementation of the AdResS algorithm is illustrated in Fig. 1; here we briefly draw the path of development of the method and provide its basic guiding principles. We suggest the reader consult the cited references for the specific details. Originally, the TR region was composed of interacting coarse-grained molecules, and the coupling with the AT region was achieved through interpolation of atomistic and coarse-grained forces via a space-dependent smoothing function in $\Delta$ (see, e.g., $[8,10])$. The $\Delta$ region is defined by the cutoff of the atomistic potential. A recent evolution of the algorithm that increases its transferability and its computational performance removes the smooth interpolation of forces and substitutes it with an abrupt interface where the molecules in $\Delta$ interact via the atomistic force field among themselves and through the coarse-grained potential with the molecules in the coarse-grained region [11]. The proper exchange of particles and the control of its thermodynamic state is assured by the application of a one-particle external force (thermodynamic force) acting on the center of mass of molecules in $\Delta$ and by a thermostat acting in the TR $+\Delta$ region. Finally, in the latest version of the code the TR region is drastically simplified and coarse-grained particles are replaced by noninteracting pointlike particles called tracers. In contrast with the previous version of the code where the interaction between the $\Delta$ region and the TR consisted of a coarse-grained potential, 
there is no interaction between the molecules of the $\Delta$ region and the tracers in the current version. In summary, the simulation box is divided into three parts: the AT region where molecules have atomistic resolution, that is, the region of physical interest; the $\Delta$ region where molecules are also represented with atomistic resolution but with the key difference being that they evolve under the additional action of the thermodynamic force and of a capping force; and the TR region where molecules are noninteracting pointlike particles whose thermal equilibrium is assured by a thermostat. The capping force acts only in the $\Delta$ region because pointlike particles coming from the TR region into the $\Delta$ region may be at unphysical distances relative to each other. As a consequence, due to the abrupt switching of molecular degrees of freedom, these molecules may experience extremely large forces between atoms, causing the simulation to crash. It must be clarified that the capping force is not a force in itself but it is a technical means by which we automatically renormalize the large forces to the average force of a fully atomistic simulation. Moreover, at the technical level, the code in the TR region treats the molecules as noninteracting entities by switching off intermolecular interactions. A water molecule leaving the $\Delta$ region and entering the TR region is mapped onto a virtual site whose trajectory corresponds to the dynamics of the tracer while the atoms of the water molecule are rigidly bound to the site and their equations of motion are no longer considered. In our previous work [7] we have shown that for a liquid of pure water at room conditions, the cooperative action of the thermodynamic force and of the thermostat assures proper equilibrium throughout the system and allows for accurate structural and dynamical properties to be calculated in the AT region. The extreme simplification of the computational setup became possible through the mapping of the algorithm onto a theoretical model of open system. Such a model fixes a series of conditions which are sufficient to assure that the physics of the AT region is correct and, in the limit of large TR and AT regions compared to $\Delta$, one has a grand-canonical-like ensemble for AT [8] (GC-AdResS). The application of the thermodynamic force in $\Delta$ is one of such conditions. This force is calculated self-consistently during the equilibration run and assures that the particle density in $\Delta$ is equal to the atomistic target density at equilibrium (see the Appendix for more details). In practice, this ensures that the chemical potential of the different regions in AdResS is set equal to the chemical potential of the fully atomistic system (see, e.g., Ref. [12]). Another condition concerns the behavior of the particle probability distribution $P(N)$ in AT; this should be the same as the $P(N)$ of the equivalent subsystem in a full atomistic simulation. Finally, one must verify that the interaction energy between molecules in AT and molecules in $\Delta$ is negligible compared to the interaction energy among the molecules in AT. As a next step, we highlight that the method can be used as a tool of analysis to define the minimal AT region required to reproduce results of a fully atomistic simulation. In essence, the method is employed to determine the minimal region where atomistic degrees of freedom are mandatory for characterizing the solvation structure of the membrane. In turn, we propose to consider such a minimal region a statistical-mechanics-based estimation of the size of the hydration shell.

\section{RESULTS}

The calculation of the thermodynamic force is made on a system of pure bulk water at the thermodynamic conditions of the solvating water in the membrane-water system and its calculation and validation are reported in the Appendix. As shown in all previous applications, given the robustness of AdResS, the thermodynamic force is transferable to the bulk water of the membrane-water system. Next, we need to determine the minimal AT region whose structural properties faithfully reproduce the structural properties calculated in the equivalent subregion of the fully atomistic system of reference. The results reported in the next subsections will show that the total AT region of minimal size is $7 \mathrm{~nm}$ corresponding to about $1.5 \mathrm{~nm}$ (on average) of hydration layer on each side of the membrane. One may argue that since atomistic degrees of freedom are also present in $\Delta$, the AT region cannot be considered as the minimal region whose atomistic degrees of freedom are necessary and sufficient to determine atomistic properties of the AT subsystem. However, the $\Delta$ region is artificial due to the application of the thermodynamic force; in $\Delta$, the atomistic molecules do not reproduce the target atomistic properties (radial distribution functions, for example), and thus, they do not support the properties of the AT region at atomistic level. For this reason, the physical action of the $\Delta$ region on the molecules of AT must be considered relevant only for macroscopic variables such as density and temperature. The results are divided into two subsections: the first concerns the verification of the mandatory conditions that define a physically meaningful open system (the AT region), and the second is a subsection where relevant structural properties, and their agreement with those of the reference fully atomistic simulation, are shown.

\section{A. Verification of mandatory conditions for a physically meaningful definition of an open system}

As discussed before, the three conditions that our adaptive setup needs to satisfy in order to define a proper open region for the AT portion of AdResS [8,10] concern (i) a basic structural property: the water density in the $\mathrm{AT}+\Delta$ region should be equal, within some numerical accuracy, to the fully atomistic reference value. This criterion assures that, by the action of the thermodynamic force in $\Delta$, one is at the same thermodynamic state point of the system of reference. (ii) $\mathrm{A}$ basic energy criterion: the interaction energy of the molecules in AT with the molecules of $\Delta$ (AT- $\Delta$ coupling energy) should be negligible compared to the interaction energy between the molecules in the AT region only (AT-AT interaction energy). This criterion assures that there are no relevant atom-atom energy contributions in AT from the reservoir. In general, this criterion is employed in statistical mechanics texts in the definitions of proper statistical, grand-canonical-like ensembles of open systems (see, e.g., [13]). Finally, (iii) a probabilistic criterion: the probability distribution function of the particle number in AT, $P(N)$, should reproduce, within a certain accuracy, the full atomistic $P(N)$ of reference. This criterion assures that the statistics (average number of molecules and average particle number fluctuation) in AT is consistent with that of the reference case. The quantitative criterion used in 


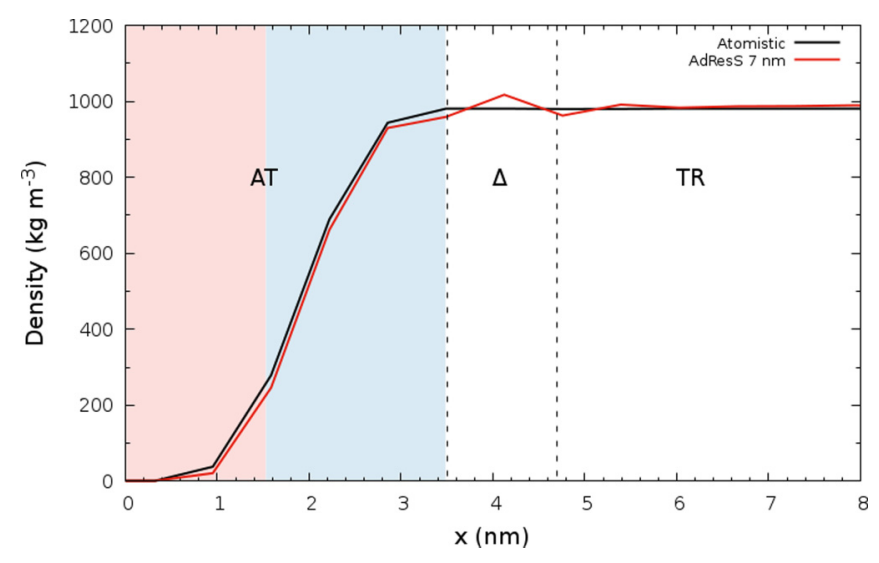

FIG. 2. Mass density profile of water in the AdResS $A T=7 \mathrm{~nm}$ and reference atomistic system. Data from both simulations has been averaged over both sides of the bilayer and symmetrized. Red shading indicates average distance of the phosphorus atoms in each leaflet from the center of the bilayer while blue shading indicates the water-DPPC interface region where density of the bilayer is still nonzero. In each case, shaded regions represent the quantities calculated in the $A T=7 \mathrm{~nm}$ AdResS simulation.

this work to define the accuracy of the results, compared to the data of reference, is $5 \%$; that is, we consider a satisfactory agreement between the AdResS data and the fully atomistic data when the discrepancy in their values does not exceed $5 \%$. Figures 2, 3, and 4 show the results obtained with AdResS and the comparison with fully atomistic results for the minimal size of the AT region, $7 \mathrm{~nm}$. For AT regions smaller than 7 $\mathrm{nm}$ in the $x$ direction, the discrepancy between the AdResS results and the reference data exceeds $5 \%$. As can be seen in Fig. 2, the agreement between AdResS and atomistic simulations is satisfactory with the largest discrepancy for the water density (reference bulk value $=980 \mathrm{~kg} \mathrm{~m}^{-3}$ ) being about $3 \%$ in the $\Delta$ region. Figure 4 shows results of calculations of the AT-AT interaction energy versus the energy associated with AT- $\Delta$ interactions. Compared to the AT-AT interaction energy, energy contributions from the $\Delta$ reservoir region in the AT subsystem are negligible (less than $2 \%$ throughout the simulation). Finally, the deviation of $P(N)$ peak values in the case of AdResS from those of the reference atomistic simulation is about $4 \%$ [Fig. 3(a)]. Shifting the $P(N)$ plots and placing them on top of one another [Fig. 3(b)] reveals a similar distribution characteristic of proper open systems further confirmed by the application of a Gaussian fit derived from the data. From such results, we can conclude that the AT region of $7 \mathrm{~nm}$ represents a physically consistent open system at the desired thermodynamic conditions. In the following subsection, we will report several representative structural properties calculated in the AdResS AT region and compare results with those calculated in the equivalent region of the fully atomistic simulation.

\section{B. Structural properties of the minimal AT region}

As representative structural properties we have chosen (i) the deuterium order parameters for the DPPC molecules and (ii) the thickness of the membrane (membrane properties); (iii) the water-water radial distribution functions (solvent
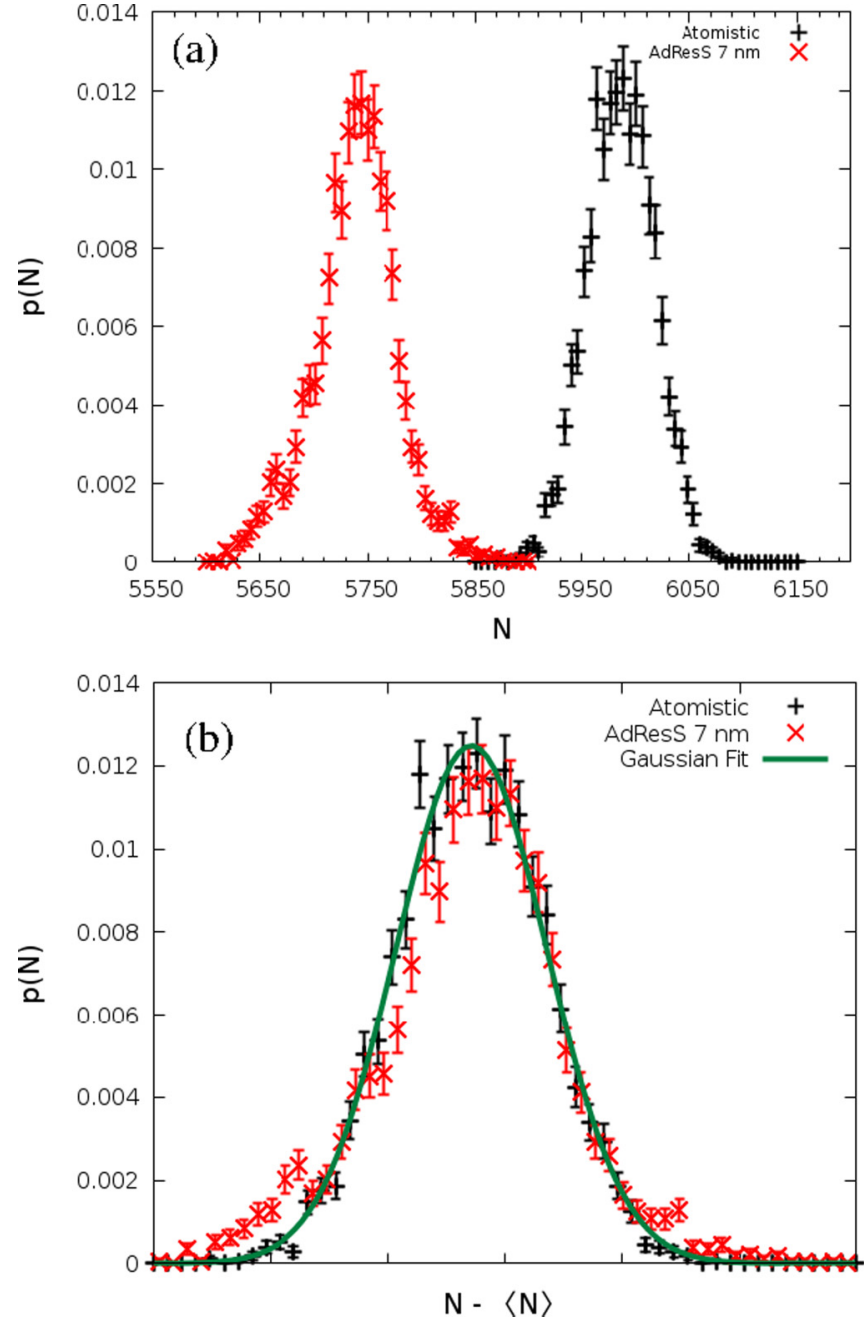

FIG. 3. (a) Probability distribution $P(N)$ of the particle number in the AdResS AT region compared with results from the equivalent subdomain of the fully atomistic reference. (b) Same data as (a), corrected for the discrepancy in peak values (about 4\%). A Gaussian fit derived from the data is applied to illustrate qualitatively the shape of both distributions.

properties); and (iv) two relevant membrane-water radial distribution functions, phosphorus-oxygen and nitrogen-oxygen (solvation structural properties).

Deuterium order parameters provide a useful measure of the disorderliness of the DPPC alkane chains that can be compared directly with the same value measured in NMR experiments. Figure 5 shows the deuterium order parameters $S_{\mathrm{CD}}$ for DPPC sn- 1 and sn- 2 hydrocarbon chains. The formula used to calculate this parameter is $S_{\mathrm{CD}}=\frac{3}{3}\left\langle\cos ^{2} \theta_{n}\right\rangle-\frac{1}{2}$, where $\theta_{n}$ is the angle between the normal to the membrane and the vector from the carbon atoms $\mathrm{C}_{n-1}$ to $\mathrm{C}_{n+1}$ along the chain. DPPC has 16 carbon atoms along each chain and the calculation considers the order parameters for carbon atoms 2 through 15 . The order parameters can vary between 1 (indicating total order along the interface normal) and $-\frac{1}{2}$ (complete order perpendicular to the normal), with a value of zero indicating uniform disorderliness. In this work, we report the absolute value of $S_{\mathrm{CD}}$. The results show very satisfactory agreement 


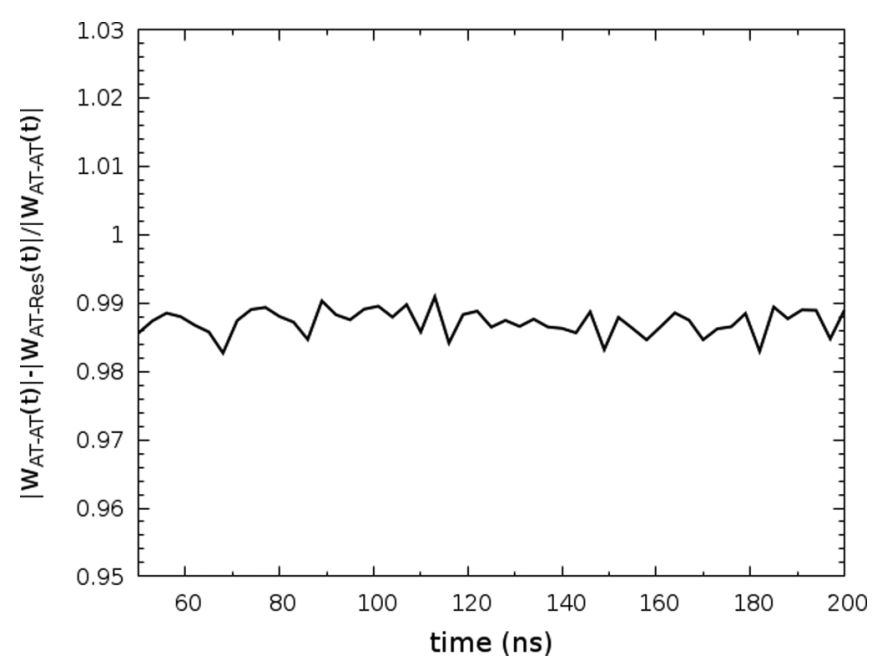

FIG. 4. Interaction energy of the AT subsystem with the reservoir relative to the AT-AT interaction as a function of time: $\frac{\left|W_{\mathrm{AT}-\mathrm{AT}}(t)\right|-\left|W_{\mathrm{AT}-\mathrm{Res}}(t)\right|}{W_{\mathrm{AT}} \text {. Th }(t)}$. The energy contribution from the reservoir in the AT subsystem is, on average, less than $2 \%$.

between the AdResS and fully atomistic data. Moreover, in each case, the trend also agrees with results from experimental data [14] and those presented in previous simulation studies [15-17]. Another representative structural property of the membrane is its average thickness; if the AdResS setup did not lead to the correct physics, one would expect artificial effects on the size and stability of the membrane and thus the thickness would exhibit visible consequences. Table I shows that the AdResS results agree with the reference results within an accuracy of about $2.7 \%$. Next, we consider radial distribution functions. Such functions represent not only specific atomistic structural signatures but their combination represents the probability distribution function of the system in configuration space up to the second order. In fact if one had the radial distribution function of each atom pair of the system, then $P\left(\mathbf{R}_{1} \ldots \mathbf{R}_{N}\right) \approx \Pi_{i, j} g\left(r_{i j}\right)$, where $\mathbf{R}_{1} \ldots \mathbf{R}_{N}$ is the configuration in space of the $N$ molecules of the system, and $i$ and $j$ label atoms belonging to different molecules at a distance $r_{i j}$. The knowledge of $P\left(\mathbf{R}_{1} \ldots \mathbf{R}_{N}\right)$ implies the capability of calculating ensemble averages of physical quantities of the system; thus, its approximation via the factorization of the radial distribution functions implies a statistical knowledge of the system up to the two-body level (second order). Here, we do not report all atom-atom radial distribution functions but only a representative sample. Figure 6 shows the oxygen-oxygen, oxygen-hydrogen, and hydrogen-hydrogen radial distribution functions calculated in the AdResS AT region compared with those calculated in the corresponding subregion of the fully atomistic system. It must be noted that the functions presented here are not normal-

TABLE I. Membrane thickness as measured from P-P distance between bilayer leaflets.

\begin{tabular}{lcc}
\hline \hline & AdResS, AT $=7 \mathrm{~nm}$ & Atomistic \\
\hline Membrane thickness & $3.94 \pm 0.2 \mathrm{~nm}$ & $4.05 \pm 0.2 \mathrm{~nm}$ \\
\hline \hline
\end{tabular}
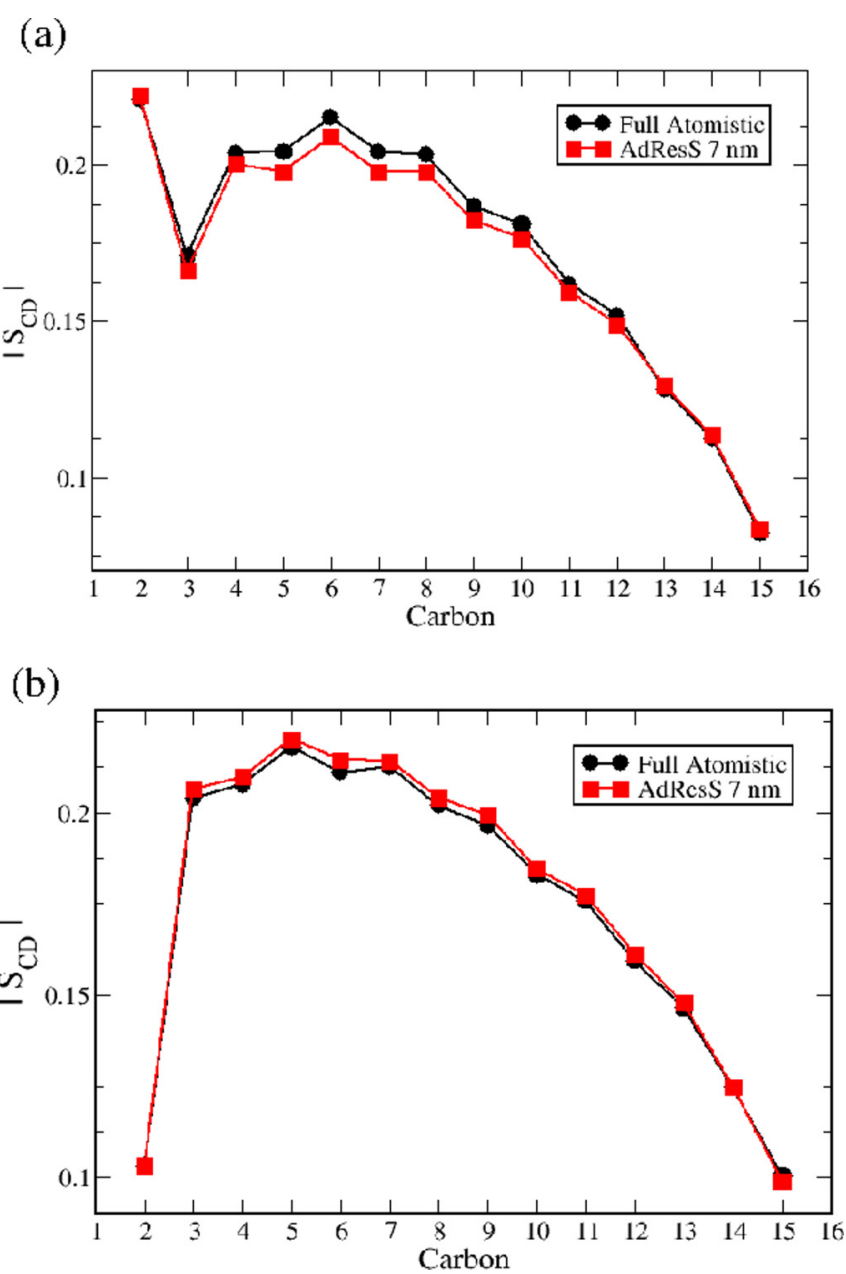

FIG. 5. Deuterium order parameters for DPPC: (a) sn-1 and (b) sn-2 acyl chains from atomistic and AdResS simulations.

ized and display a decreasing behavior for large distances; this is due to their calculation in a subregion of a larger system. Here, the relevant message concerns the agreement between the AdResS and reference simulations, and their agreement is highly satisfactory. The same satisfactory agreement can be seen for the phosphorus-oxygen and nitrogenoxygen radial distribution functions chosen as examples for the characterization of the spatial correlations between solvating water molecules and lipid head groups, as reported in Fig. 7.

\section{Comparison with available data in the literature}

The intuitive idea of the hydration shell considers the first few water layers surrounding a biomolecule which are noticeably influenced by it. On a more quantitative level, in current literature the hydration shell is often defined by a distance up to where the aqueous environment, sufficiently far from the biomolecular surface, presents bulklike water behavior; that is, the distance is defined by the first minimum of radial distribution functions or by the distance where the water density assumes a bulk profile [2,3,18]. Either way, none of these definitions can assure the fulfillment of the criterion based on the "influence of the biomolecule on the 

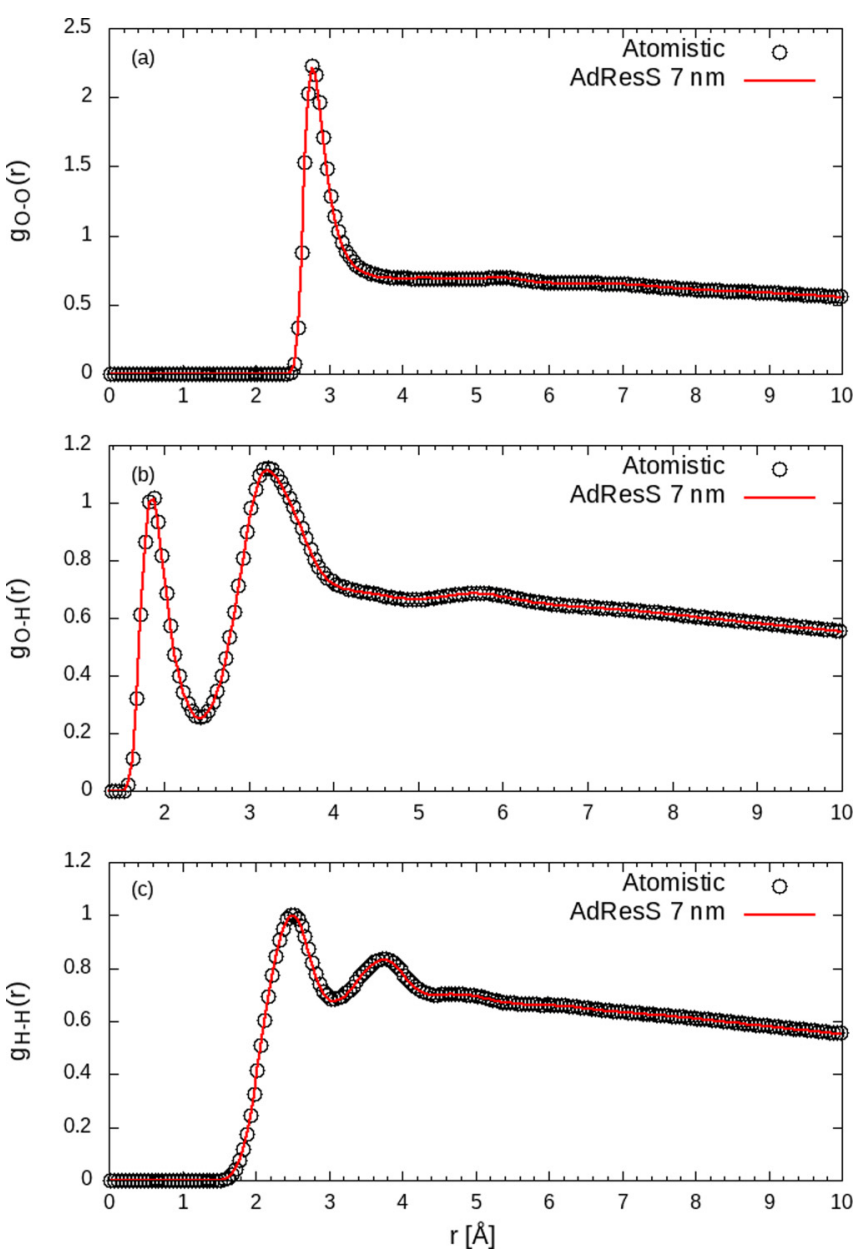

FIG. 6. Comparison of (a) oxygen-oxygen, (b) oxygenhydrogen, and (c) hydrogen-hydrogen water-water radial distribution functions from the AT AdResS region and the equivalent subsystem from the atomistic simulation of reference. The functions are not normalized.

water behavior." The criterion suggested by this work naturally takes into account the influence of the membrane on the atomistically resolved structural properties of water, and thus on the specific details of the hydration structure. An interesting point is that if one applies the geometric criterion of the first minimum of the DPPC-water radial distribution functions of Fig. 7, we would get a much smaller size for the hydration shell, below the $1.5 \mathrm{~nm}$ found by us. Results similar to ours are also found in direct determination of the hydration layer around biomolecules using spectroscopic and molecular dynamics data. For example, in a recent paper by Charkhest and coworkers [19], it was shown that a protein (bovine serum albumin) influences the dynamics of water well beyond the first solvation layer, to around $0.7 \mathrm{~nm}$ from the protein surface. In conclusion, while we certainly cannot claim that our criteria of analysis represents a universal definition of hydration shell, our technique can certainly be employed in a complementary way to identify relevant degrees of freedom involved in the hydration process and be used in relation to findings of other groups that employ other tools of analysis for a proper interpretation of hydration mechanisms.
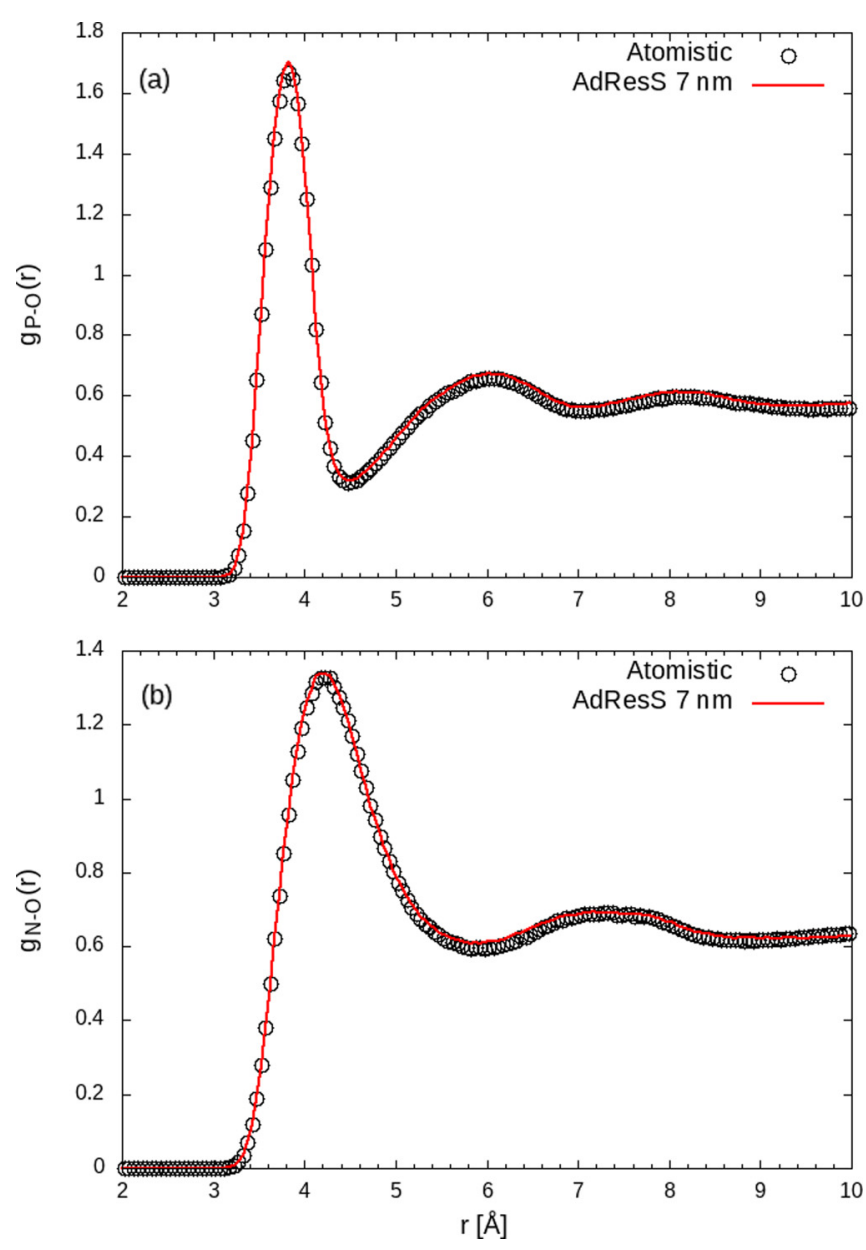

FIG. 7. Comparison of (a) phosphorus-oxygen and (b) nitrogenoxygen DPPC-water radial distribution functions from the AT AdResS region and the equivalent subsystem from the atomistic simulation of reference. The functions are not normalized.

\section{CONCLUSIONS}

We have shown that the AdResS method in its latest version, where the reservoir is composed of noninteracting point particles (tracers) with a direct interface to the atomistic molecules, can satisfactorily describe a highly complex system such as the hydrated biological membrane treated in this work. The full atomistic simulation of reference of this work was calibrated by reproducing state of the art simulations of similar systems available in the literature [20]. Since the AdResS method is based on stringent criteria in the definition of a physically consistent subsystem embedded in a large reservoir, the minimal atomistic region considered in this work represents a physically meaningful subsystem where structural properties are defined solely by the atomistic degrees of freedom of this region. Such a feature of AdResS suggests that one can estimate the hydration shell of the membrane based on the statistical definition of the minimal number of relevant atomistic degrees of freedom of the solvating water, that is, the number of water molecules in the smallest possible atomistic region of AdResS. We have proposed this idea and verified numerically that several representative structural properties in the atomistic region of 
AdResS, with a size of $7.0 \mathrm{~nm}$ (corresponding to about $1.5 \mathrm{~nm}$ for the hydration shell), faithfully reproduce (with an accuracy higher than 5\%) the structural properties the system would have if one considers a fully atomistic simulation of reference. The estimate of the size of the hydration shell proposed here complements other definitions available in the literature [2] and in particular may be helpful to estimate the region where the change of chemical degrees of freedom (i.e., atomistic degrees of freedom) can have an immediate impact on the hydration structures and processes.

\section{ACKNOWLEDGMENTS}

This research has been funded by Deutsche Forschungsgemeinschaft (DFG) through Grant No. CRC 1114: "Scaling Cascades in Complex Systems," Project No. C01, and Grant No. DE 1140/7-3. The simulations were performed with the HPC resources provided by the North-German Supercomputing Alliance (HLRN), Project No. bec00203.

\section{APPENDIX: TECHNICAL DETAILS}

\section{System}

A fully atomistic lipid bilayer composed of 180 (90 per leaflet) DPPC molecules was constructed using the CHARMM-GUI membrane builder tool [21]. The bilayer was then solvated with 52470 molecules of the modified CHARMM-TIP3P [22] water model in a box of initial dimensions $33.4 \mathrm{~nm} \times 7.5 \mathrm{~nm} \times 7.5 \mathrm{~nm}$. The membrane was oriented in the $y-z$ plane with its normal along the $x$ axis.

\section{Atomistic simulation details}

All reference atomistic simulations were performed with the CHARMM36 [15] force field using GROMACS 5.1 [23] at a time step of $2 \mathrm{fs}$, achieved through the use of the LINCS algorithm [24]. Prior to the production run, the energy of the solvated membrane system was minimized using the steepest descents method, then equilibrated in the $N V T$ ensemble for $500 \mathrm{ps}$, followed by a $4 \mathrm{~ns} N P T$ equilibration using a leapfrog integrator. $N V T$ equilibration was performed using the Berendsen thermostat with a coupling constant of $1.0 \mathrm{ps}$. Pressure was maintained at 1 bar during $N P T$ equilibration with semi-isotropic Berendsen pressure coupling with a coupling constant of $5.0 \mathrm{ps}$. Following this, the system was again equilibrated in the $N P T$ ensemble using a stochastic dynamics integrator with a coupling constant of $0.1 \mathrm{ps}$ and the semiisotropic Parrinello-Rahman barostat with a coupling constant of $5.0 \mathrm{ps}$ for a total of $100 \mathrm{~ns}$. This phase was considered complete when the box dimensions converged to relatively constant average values and thus, area per lipid values were also constant $\left(A_{L} \approx 63 \AA^{2} / \mathrm{DPPC}\right.$, in accordance with experimental values [25]). At this point, pressure coupling was switched off, box vectors were set at their average values from the previous $N P T$ phase $(31.71740 \mathrm{~nm} \times 7.57654 \mathrm{~nm} \times$ $7.57654 \mathrm{~nm}$ ), and data were collected in the $N V T$ ensemble for $250 \mathrm{~ns}$. The temperature in all cases was maintained at $323.15 \mathrm{~K}$ (above the liquid-disordered phase transition temperature for DPPC) and the membrane and water were coupled separately to individual thermostats. Periodic bound- ary conditions were applied in all directions and the particle mesh Ewald [26] method was applied for calculating longrange electrostatic interactions. Dispersion correction was not applied. All further simulation parameters follow the recommended, optimized protocol found in Ref. [20]. Namely, this involves using a force-switch cutoff beginning at $1.0 \mathrm{~nm}$ and ending at $1.2 \mathrm{~nm}$ in order to ensure accurate truncation of the van der Waals interactions.

\section{AdResS simulation details}

The AdResS setup was built from the same box used for the reference atomistic $N V T$ production simulation detailed above. Simulations were performed with the newest AdResS methodology detailed in Ref. [7] with a modified GROMACS 5.1. The simulation box is divided along the $x$ axis in one AT region, two $\Delta$ (transition) regions, and two TR regions which are connected through periodic boundary conditions. Five separate simulations were performed with the AT region varying in length from $10 \mathrm{~nm}$ to $6 \mathrm{~nm}$ in increments of $1 \mathrm{~nm}$. The length of each $\Delta$ region is fixed at $1.2 \mathrm{~nm}$ regardless of the AT size, and the TR region size varies according to the size of the AT region. In each simulation, data were collected for $250 \mathrm{~ns}$ in the $N V T$ ensemble. AdResS simulations used the same parameters as the reference atomistic $N V T$ simulation with the exception of electrostatic calculations, which utilized the reaction-field method, as in all previous AdResS studies, and the coupling constant of the stochastic dynamics integrator, which was set to $0.01 \mathrm{ps}$.

\section{Calculation and validation of the thermodynamic force}

\section{a. Atomistic simulation of reference}

In order to have a point of comparison to gauge the validity of our thermodynamic force $\left[F_{\text {th }}(x)\right]$ calculation, a fully atomistic simulation of reference was initially prepared with a simulation box of dimensions $33.2 \mathrm{~nm} \times 7.4 \mathrm{~nm} \times 7.4 \mathrm{~nm}$ filled with 58990 CHARMM-TIP3P water molecules. The system was subjected to energy minimization via the steepest descents method and then equilibrated in the NPT ensemble in two phases. First, a 500 ps simulation was performed using a leap-frog integrator, the Berendsen thermostat with a coupling constant of $1.0 \mathrm{ps}$, and the Berendsen barostat with a coupling constant of $5.0 \mathrm{ps}$ applied isotropically. For the second phase, we continued the previous simulation for $2 \mathrm{~ns}$, switching to a stochastic integrator with a coupling constant of $0.1 \mathrm{ps}$ and the Parrinello-Rahman barostat with a coupling constant of $5.0 \mathrm{ps}$ applied isotropically. Following these NPT equilibrations, the box vectors were set to their average values for a final simulation box size of dimensions $33.096 \mathrm{~nm} \times$ $7.37682 \mathrm{~nm} \times 7.37682 \mathrm{~nm}$. From this point, the system was equilibrated further in the $N V T$ ensemble for $3.0 \mathrm{~ns}$, again utilizing a stochastic integrator with a coupling constant of 0.1 ps. Finally, a production $N V T$ simulation of $40 \mathrm{~ns}$ was performed using the same parameters as the previous $N V T$ equilibration. In all cases, all other parameters are identical to those listed in A 2 of this Appendix. 

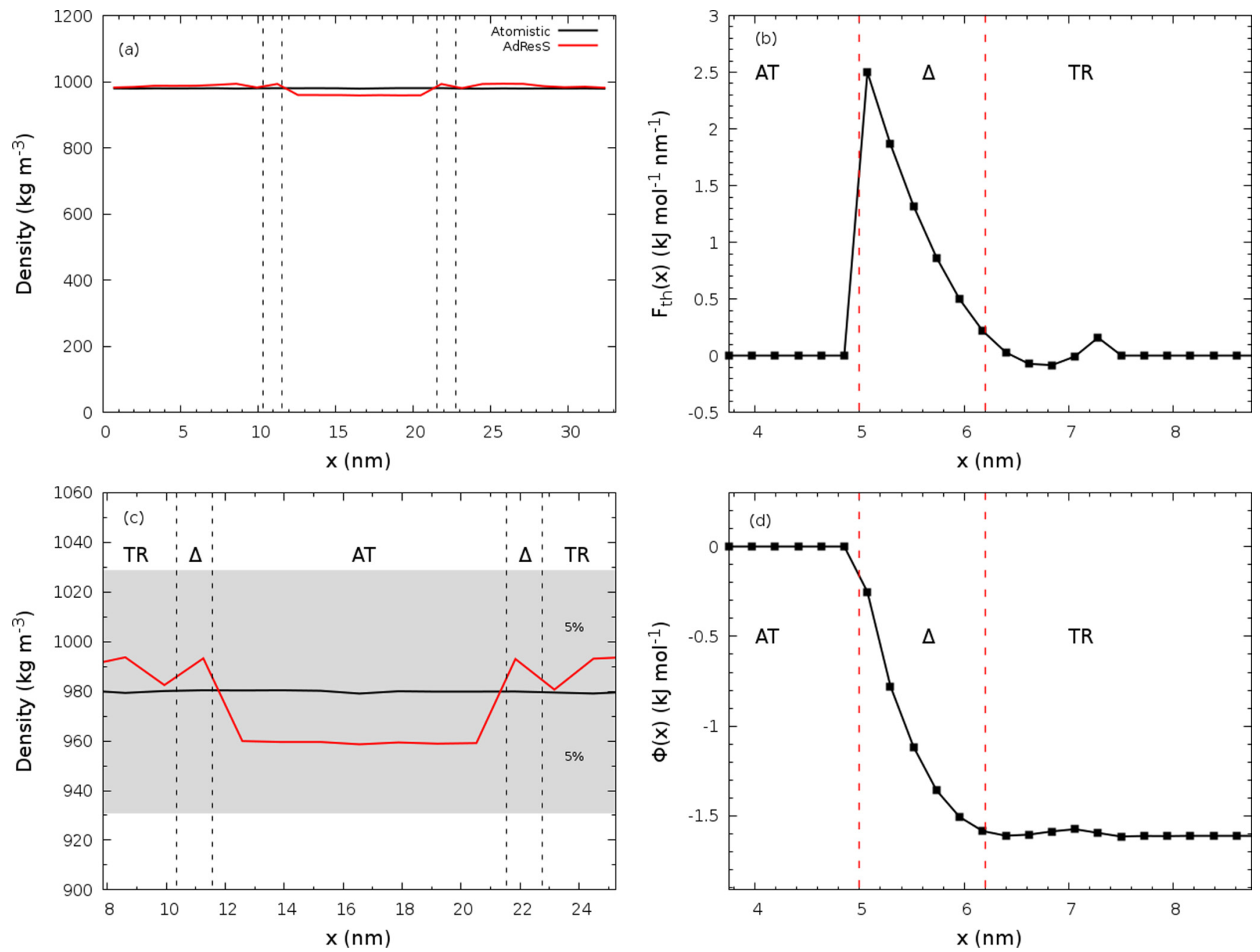

FIG. 8. (a) Mass density of the atomistic reference system and AdResS system calculated across the entire box in the $x$ direction. Dashed lines indicate the borders of the $\Delta$ transition regions. (b) The thermodynamic force $F_{\text {th }}(x)$ along the $x$ axis. By construction, $F_{\text {th }}(x)=0$ in the AT region. (c) A zoomed-in view of (a) giving a clearer view of the atomistic reference density and the density facilitated by $F_{\text {th }}(x)$ in the AdResS simulation. The gray band indicates our allowed accuracy goal of at most $5 \%$ deviation from any calculated atomistic property. (d) The integral of $F_{\mathrm{th}}(x)$, the thermodynamic potential $\Phi_{\mathrm{th}}(x)$.

\section{b. Calculation of the thermodynamic force}

The thermodynamic force was calculated for a system of pure bulk water, in a procedure similar to that of our previous paper [7]. The same simulation box from the atomistic reference simulation was used and split up into an AT region of $x=10 \mathrm{~nm}$ flanked by $\Delta$ regions of $x=1.2 \mathrm{~nm}$ each with the rest of the box in the $x$ direction represented by tracer particles. AdResS simulation parameters are identical to those given in A 3 of this Appendix. The thermodynamic force was calculated iteratively as $F_{\text {th }}^{i+1}(x)=F_{\text {th }}^{i}(x)-\frac{M_{\alpha}}{\left[\rho_{\text {ref }}\right]^{2} \kappa} \nabla \rho^{i}(x), M_{\alpha}$ is the mass of the molecule, $\kappa$ a (conveniently) tunable constant determined from all previous tests with the application of AdResS, $\rho^{i}(x)$ is the molecular density as a function of the position in $\Delta$ at the $i$ th iteration, and $\rho_{\text {ref }} \equiv \rho_{\text {AdResS }}$ is the density of reference of the full atomistic system. The convergence criterion depends on the accuracy required for the simulation, but following the experience, $\left|\rho_{\text {AdResS }}-\rho_{\text {atomistic }}\right|$ should be always below $5 \%$ in $\Delta$. At each iteration step
$F_{\text {th }}^{i+1}(x)$ was calculated employing cubic splines parametrized on a uniform grid with spacing of $0.22064 \mathrm{~nm}$. It was obtained over ten $1 \mathrm{~ns}$ iterations and was considered converged when $\left|\rho_{\text {atomistic }}(x)-\rho_{\text {AdResS }}(x)\right| / \rho_{\text {atomistic }} \approx 2 \%$.

\section{c. Validation of thermodynamic force}

Following the calculation of $F_{\text {th }}(x)$, it was used to perform a $40 \mathrm{~ns}$ production simulation with a setup identical to that given above. Subsequently, several thermodynamic and structural properties were calculated and compared with those of the reference atomistic simulation. The mass density in the $x$ direction, the probability distribution of the number density of water molecules in the AT region, and $\mathrm{O}-\mathrm{O}, \mathrm{O}-\mathrm{H}$, and $\mathrm{H}-\mathrm{H}$ radial distribution functions were computed and were found to be within $\approx 2 \%$ of those of the reference. Figure 8 presents a comparison between the mass density in the AdResS and atomistic simulations as well as the $F_{\text {th }}(x)$ and its integral, the thermodynamic potential $\Phi_{\mathrm{th}}(x)$. 
[1] E. Disalvo, F. Lairion, F. Martini, E. Tymczyszyn, M. Frías, H. Almaleck, and G. Gordillo, Structural and functional properties of hydration and confined water in membrane interfaces, Biochim. Biophys. Acta, Biomembr. 1778, 2655 (2008).

[2] D. Laage, T. Elsaesser, and J. Hynes, Water dynamics in the hydration shells of biomolecules, Chem. Rev. 117, 10694 (2017).

[3] E. Duboue-Dijon, A. Fogarty, J. Hynes, and D. Laage, Dynamical disorder in the DNA hydration shell, J. Am. Chem. Soc. 138, 7610 (2016).

[4] B. Shadrack Jabes and L. Delle Site, Nanoscale domains in ionic liquids: A statistical mechanics definition for molecular dynamics studies, J. Chem. Phys. 149, 184502 (2018).

[5] B. P. Lambeth, C. Junghans, K. Kremer, C. Clementi, and L. Delle Site, On the locality of hydrogen bond networks at hydrophobic interfaces, J. Chem. Phys. 133, 221101 (2010).

[6] B. Shadrack Jabes, R. Klein, and L. Delle Site, Structural locality and early stage of aggregation of micelles in water: An adaptive resolution molecular dynamics study, Adv. Theor. Simul. 1, 1800025 (2018).

[7] L. Delle Site, C. Krekeler, J. Whittaker, A. Agarwal, R. Klein, and F. Höfling, Molecular dynamics of open systems: Construction of a mean-field particle reservoir, Adv. Theor. Simul. 2, 1900014 (2019).

[8] G. Ciccotti and L. Delle Site, The physics of open systems for the simulation of complex molecular environments in soft matter, Soft Matter 15, 2114 (2019).

[9] T. Tarenzi, V. Calandrini, R. Potestio, and P. Carloni, Openboundary molecular mechanics/coarse-grained framework for simulations of low-resolution G-protein-coupled receptorligand complexes, J. Chem. Theory Comput. 15, 2101 (2019).

[10] L. Delle Site and M. Praprotnik, Molecular systems with open boundaries: Theory and simulation, Phys. Rep. 693, 1 (2017).

[11] C. Krekeler, A. Agarwal, C. Junghans, M. Praprotnik, and L. Delle Site, Adaptive resolution molecular dynamics technique: Down to the essential, J. Chem. Phys. 149, 024104 (2018).

[12] H. Wang, C. Hartmann, C. Schütte, and L. Delle Site, GrandCanonical-Like Molecular-Dynamics Simulations by Using an Adaptive-Resolution Technique, Phys. Rev. X 3, 011018 (2013).

[13] K. Huang, Statistical Mechanics, 2nd ed. (John Wiley \& Sons, Inc., New York, 1987).

[14] J. Douliez, A. Léonard, and E. Dufourc, Restatement of order parameters in biomembranes: Calculation of $\mathrm{C}-\mathrm{C}$ bond order parameters from C-D quadrupolar splittings, Biophys. J. 68, 1727 (1995).

[15] J. Klauda, R. Venable, J. Freites, J. W. O’Connor, D. Tobias, C. Mondragon-Ramirez, I. Vorobyov, A. MacKerell, and R. Pastor, Update of the CHARMM all-atom additive force field for lipids: Validation on six lipid types, J. Phys. Chem. B 114, 7830 (2010).

[16] A. Kukol, Lipid models for united-atom molecular dynamics simulations of proteins, J. Chem. Theory Comput. 5, 615 (2009).

[17] M. Patra, M. Karttunen, M. Hyvönen, E. Falck, P. Lindqvist, and I. Vattulainen, Molecular dynamics simulations of lipid bilayers: Major artifacts due to truncating electrostatic interactions, Biophys. J. 84, 3636 (2003).

[18] G. Stirnemann, E. Wernersson, P. Jungwirth, and D. Laage, Mechanisms of acceleration and retardation of water dynamics by ions, J. Am. Chem. Soc. 135, 11824 (2013).

[19] A. Charkhesht, C. Regmi, K. Mitchell-Koch, S. Cheng, and N. Q. Vinh, High-precision megahertz-to-terahertz dielectric spectroscopy of protein collective motions and hydration dynamics, J. Phys. Chem. B 122, 24 (2018).

[20] J. Lee, X. Cheng, J. Swails, P. Yeom, M. S. Eastman, J. Lemkul, S. Wei, J. Buckner, J. Jeong, Y. Qi, S. Jo, V. Pande, D. Case, C. Brooks, A. MacKerell, J. Klauda, and W. Im, CHARMM-GUI input generator for NAMD, GROMACS, AMBER, OpenMM, and CHARMM/OpenMM simulations using the CHARMM36 additive force field, J. Chem. Theory Comput. 12, 405 (2016).

[21] S. Jo, T. Kim, V. G. Iyer, and W. Im, CHARMM-GUI: A web-based graphical user interface for CHARMM, J. Comput. Chem. 29, 1859 (2008).

[22] A. D. MacKerell, D. Bashford, M. Bellott, R. L. Dunbrack, J. D. Evanseck, M. J. Field, S. Fischer, J. Gao, H. Guo, S. Ha, D. Joseph-McCarthy, L. Kuchnir, K. Kuczera, F. T. K. Lau, C. Mattos, S. Michnick, T. Ngo, D. T. Nguyen, B. Prodhom, W. E. Reiher, B. Roux, M. Schlenkrich, J. C. Smith, R. Stote, J. Straub, M. Watanabe, J. Wiórkiewicz-Kuczera, D. Yin, and M. Karplus, All-atom empirical potential for molecular modeling and dynamics studies of proteins, J. Phys. Chem. B 102, 3586 (1998).

[23] M. J. Abraham, T. Murtola, R. Schulz, S. Pall, J. Smith, B. Hess, and E. Lindahl, GROMACS: High performance molecular simulations through multi-level parallelism from laptops to supercomputers, Software X 1-2, 19 (2015).

[24] B. Hess, H. Bekker, H. J. C. Berendsen, and J. E. M. Fraaije, LINCS: A linear constraint solver for molecular simulations, J. Comput. Chem. 18, 1463 (1997).

[25] N. Kučerka, M.-P. Nieh, and J. Katsaras, Fluid phase lipid areas and bilayer thicknesses of commonly used phosphatidylcholines as a function of temperature, Biochim. Biophys. Acta, Biomembr. 1808, 2761 (2011).

[26] T. Darden, D. York, and L. Pedersen, Particle mesh Ewald: An $N \cdot \log (N)$ method for Ewald sums in large systems, J. Chem. Phys. 98, 10089 (1993). 